\title{
Supporting sequences of pure states on JB algebras
}

by

\author{
JAN HAMHALTER (Praha)
}

Abstract. We show that any sequence $\left(\varphi_{n}\right)$ of mutually orthogonal pure states on a JB algebra $A$ such that $\left(\varphi_{n}\right)$ forms an almost discrete sequence in the relative topology induced by the primitive ideal space of $A$ admits a sequence $\left(a_{n}\right)$ consisting of positive, norm one, elements of $A$ with pairwise orthogonal supports which is supporting for $\left(\varphi_{n}\right)$ in the sense of $\varphi_{n}\left(a_{n}\right)=1$ for all $n$. Moreover, if $A$ is separable then $\left(a_{n}\right)$ can be taken such that $\left(\varphi_{n}\right)$ is uniquely determined by the biorthogonality condition $\varphi_{n}\left(a_{n}\right)=1$. Consequences of this result improving hitherto known extension theorems for $C^{*}$-algebras and descriptions of dual JB algebras are given.

1. Introduction and preliminaries. Let $\left(x_{n}\right)$ be a sequence of points in a metrizable compact Hausdorff space $X$ such that $\left(x_{n}\right)$ is a discrete space in its relative topology. It is well known that there is a "dual" supporting sequence $\left(f_{n}\right)$ consisting of positive, norm one, continuous functions on $X$ with pairwise orthogonal supports and such that $\left(x_{n}\right)$ is the only sequence in $X$ with $f_{n}\left(x_{n}\right)=1$ for all $n$. The aim of this note is to study possible extensions of this fact to the "non-associative" generalization of compact spaces given by the Jordan operator algebras and to explore their consequences. In this context the question concerning existence of supporting sequence translates as follows. Let $\left(\varphi_{n}\right)$ be a sequence of pure states on a separable JB algebra $A$ which are pairwise orthogonal, i.e. $\left\|\varphi_{n}-\varphi_{m}\right\|=2$ whenever $n \neq m$. Is there a sequence $\left(a_{n}\right)$ of pairwise orthogonal, norm one, positive elements of $A$ such that $\left(\varphi_{n}\right)$ is the only sequence of states on $A$ satisfying $\varphi_{n}\left(a_{n}\right)=1$ for all $n$ ? We show that the answer is affirmative under an obvious restriction on the position of states.

The concept of supporting sequence is relevant to the question of the relationship between pure states and abelian subalgebras. This problem has been deeply studied in the setting of $C^{*}$-algebras so far $[1-6,9,10,12]$. In particular, it has been proved in [2] that for any finite family $\varphi_{1}, \ldots, \varphi_{n}$ of orthogonal pure states on a separable $C^{*}$-algebra $A$ there is a maximal abelian subalgebra $B$ of $A$ such that all the states $\varphi_{1}, \ldots, \varphi_{n}$ are unique

1991 Mathematics Subject Classification: 46L30, 46L70, 46L60, 81P10. 
extensions of pure states on $B$. By using our technique of biorthogonal determining sequences we generalize this result, both by proving its validity for $\mathrm{JB}$ algebras and by showing that $B$ can be taken finitely generated. Moreover, a stronger version of this result involving infinitely many pure states is obtained (compare $[3,5]$ ). In the case of infinitely many states we have to assume that the given states are separated as points in the primitive ideal space. The necessity of this assumption is illustrated in the concluding part of the paper, where the dual JB algebras are characterized as being precisely those for which any system of pairwise orthogonal pure states admits a determining supporting system. It follows e.g. that a separable JB algebra is dual if and only if it is closed with respect to decreasing sequences of operator commuting positive elements. This, together with other results obtained, extends hitherto known characterizations of dual algebras by means of their intrinsic properties [7].

The problems studied in this paper stem also naturally from the operatoralgebraic approach to quantum theory, where observables of a quantum systems are given by JB algebras and "phase spaces" are modelled by their pure state spaces (see e.g. [13]). In this framework our results can be expressed briefly by saying that any sequence of mutually exclusive states of the quantum system is uniquely determined by some (smallest possible) classical (i.e. associative) subsystem. This contributes to the theory of hidden variables (see e.g. [8]).

Let us now recall a few notions and fix the notation. A $J B$ algebra $(A, 0)$ is a real Banach algebra for which the norm obeys

$$
\|a \circ b\| \leq\|a\| \cdot\|b\|, \quad\left\|a^{2}-b^{2}\right\| \leq \max \left(\|a\|^{2},\|b\|^{2}\right), \quad\left\|a^{2}\right\|=\|a\|^{2} .
$$

(For all unmentioned details on JB algebras we refer to [11].) Throughout the paper $A$ will always stand for a JB algebra and $A^{+}$for its positive part (i.e. $A^{+}=\left\{a^{2} \mid a \in A\right\}$ ). The order on $A$ is given by the positive cone $A^{+}$. For a fixed $a \in A$ the mappings $T_{a}, U_{a}: A \rightarrow A$ are defined by putting

$$
T_{a}(b)=a \circ b, \quad U_{a}(b)=2 a \circ(a \circ b)-a^{2} \circ b .
$$

The elements $a, b \in A$ are said to be operator commuting if $T_{a} T_{b}=T_{b} T_{a}$. A subspace $I \subset A$ is called a quadratic (resp. Jordan) ideal if $U_{a}(A) \subset I$ (resp. $T_{a}(A) \subset I$ ) for all $a \in I$. A JB algebra is said to be a JBW algebra if it has a predual. The second dual $A^{* *}$ of $A$ is a JBW algebra whose product is separately weak*-continuous and extends the original product of $A$.

In the sequel $A$ will always be considered as a weak*-dense subalgebra of $A^{* *}$. For any set $S \subset A^{* *}$ the symbol $\bar{S}$ denotes its weak*-closure. If $I$ is a Jordan ideal in $A$ then $\bar{I}=c(I) A^{* *}$, where $c(I)$ is the uniquely determined central projection in $A^{* *}$. A projection in $A^{* *}$ is said to be open if it is the supremum of some increasing net of elements of $A$. The range projection $r(a)$ of an element $a \in A^{* *}$ is defined as the infimum of the set of all projections $p \in A^{* *}$ for which $p \circ a=a$. Recall that $r(a)$ is open whenever $a \in A$. Elements $a, b \in A$ are said to be orthogonal if $a \circ b=0$, or equivalently, if $r(a) \circ r(b)=0$.

Any representation $\pi$ of $A$ into some JBW algebra $M$ extends uniquely to a. normal representation (denoted again by $\pi$ ) of $A^{* *}$ into $M$. We denote by $c(\pi)$ the central projection in $A^{* *}$ uniquely given by $\operatorname{Ker} \pi=(1-c(\pi)) A^{* * *}$. The representation $\pi$ is a factor representation if and only if $c(\pi)$ is a minimal projection in the center of $A^{* *}$. Recall that two factor representations $\pi_{1}$ and $\pi_{2}$ are equivalent if and only if $c\left(\pi_{1}\right)=c\left(\pi_{2}\right)$. The state on $A$ is a positive, norm one, linear functional. The state space $S(A)$ of $A$ is the convex set consisting of all states on $A$, and the pure state space $P(A)$ is the set of all extreme points of $S(A)$. The elements of $P(A)$ are called pure states. For each pure state $\varphi$ on $A$ there is a uniquely determined minimal projection $s(\varphi)$ in $A^{* *}$ such that $U_{s(\varphi)}(a)=\varphi(a) s(\varphi)$ for all $a \in A$. We call $s(\varphi)$ the support projection of $\varphi$. For the left kernel $\mathcal{L}_{\varphi}=\left\{a \in A \mid \varphi\left(a^{2}\right)=0\right\}$ of a pure state $\varphi$ we then have $\mathcal{L}_{\varphi}=U_{1-s(\varphi)}\left(A^{* *}\right) \cap A$. Two pure states $\varphi$ and $\varrho$ are called orthogonal if $s(\varphi) \circ s(\varrho)=0$ or, what is the same, if $\|\varphi-\varrho\|=2$. Further, let $c(\varphi)$ denote the minimal projection in the center of $A^{* *}$ majorizing the support projection $s(\varphi)$ of a pure state $\varphi$. Then $c(\varphi) A^{* *}$ is a JBW factor of type I and the mapping $\pi_{\varphi}: A \rightarrow c(\varphi) A^{* *}: a \mapsto c(\varphi) \circ a$ is a representation of $A$ onto a weak*-dense subalgebra of $c(\varphi) A^{* *}$. We call $\pi_{\varphi}$ the representation corresponding to $\varphi$. Recall that $c\left(\pi_{\varphi}\right)=c(\varphi)$. Pure states $\varphi$ and $\varrho$ are called equivalent if $c(\varphi)=c(\varrho)$.

Finally, the primitive ideal space of $A$ is defined to be the set $\operatorname{Prim}(A)=$ $\left\{\operatorname{Ker} \pi_{\varphi} \mid \varphi \in P(A)\right\}$. The set $\operatorname{Prim}(A)$ is topologized by the Jacobson topology which is given by the closure operation $S \mapsto \bar{S}=\{t \in \operatorname{Prim}(A) \mid t \supset$ $\left.\bigcap_{s \in S} s\right\}$. The symbol $\tau$ will always stand for the canonical mapping $\tau: \varphi \mapsto$ $\operatorname{Ker} \pi_{\varphi}$ of $P(A)$ onto $\operatorname{Prim}(A)$.

2. Supporting systems, In this section we deal with the existence of supporting sequences. Let $\left(\varphi_{\alpha}\right)$ be a system of mutually orthogonal pure states on $A$. We say that a system $\left(a_{\alpha}\right)$ of positive, norm one, pairwise orthogonal elements of $A$ is supporting for $\left(\varphi_{\alpha}\right)$ if $\varphi_{\alpha}\left(a_{\alpha}\right)=1$ for all $\alpha$. Our first goal is to establish the existence of supporting systems for finitely many pure states. For this we need the following auxiliary lemmas. We write $M_{3}^{8}$ for the algebra of all hermitian $3 \times 3$ matrices over Cayley numbers.

2.1. LEMMA. Suppose $\pi_{1}$ and $\pi_{2}$ are non-zero representations of $A$ with $\pi_{1}\left(A^{* *}\right)=M_{1}$ and $\pi_{2}\left(A^{* *}\right)=M_{2}$, where $M_{1}$ is a finite sum of copies of $M_{3}^{8}$ and $M_{2}$ is a JC algebra. Then neither $\operatorname{Ker} \pi_{1} \subset \operatorname{Ker} \pi_{2}$ nor $\operatorname{Ker} \pi_{2} \subset \operatorname{Ker} \pi_{1}$. 
Proof. Suppose, for a contradiction, that $\operatorname{Ker} \pi_{2} \subset \operatorname{Ker} \pi_{1}$. Then $\pi_{1}$ gives rise to a representation of the quotient $A / \operatorname{Ker} \pi_{2} \simeq \pi_{2}(A) \subset M_{2}$ onto $M_{1}$. This means that the JC algebra $\pi_{2}(A)$ has a representation onto $M_{3}^{8}$ contradicting the fact that any quotient of a JC algebra has to be a JC algebra again [11, Prop. 4.7.4, p. 118].

Similarly, if $\operatorname{Ker} \pi_{1}$ were contained in $\operatorname{Ker} \pi_{2}$ then $M_{1}$ would admit a quotient isomorphic to a non-zero JC algebra, which is a contradiction because $M_{1}$ is purely exceptional. The proof is complete.

2.2. LEMMA. Let $\pi=\pi_{1} \oplus \ldots \oplus \pi_{n}$, where $\pi_{1}, \ldots, \pi_{n}$ are inequivalent factor representations of $A$. Let $I$ be a Jordan ideal in $A$ such that each $\pi_{i}$ is non-zero on $I$. Then

$$
\overline{\pi(A)}=\overline{\pi(I)} .
$$

Proof. Without loss of generality, we can assume that $\pi_{i}(a)=c\left(\pi_{i}\right) \circ a$ for each $a \in A$. As $\pi_{i} \mid I \neq 0$ and $c\left(\pi_{i}\right)$ is a minimal central projection in $A^{* *}$ we have $c\left(\pi_{i}\right) \leq c(I)$. Since

$$
\overline{\pi(A)}=\overline{\pi_{1}(A)} \oplus \ldots \oplus \overline{\pi_{n}(A)}
$$

by inequivalence we get

$$
\overline{\pi(A)}=\sum_{i=1}^{n} c\left(\pi_{i}\right) A^{* *}=\sum_{i=1}^{n} c\left(\pi_{i}\right) c(I) A^{* *}=\sum_{i=1}^{n} c\left(\pi_{i}\right) \bar{I}=\overline{\pi(I)} .
$$

The proof is complete.

2.3. Proposition. Any finite sequence $\varphi_{1}, \ldots, \varphi_{n}$ of mutually orthogonal pure states on $A$ admits a supporting sequence.

Proof. Denote by $\pi_{i}$ the type I factor representation corresponding to $\varphi_{i}$. By the structure theory of JBW algebras, $\overline{\pi_{i}(A)}=c\left(\varphi_{i}\right) A^{* * *}$ is isomorphic either to $M_{3}^{8}$ or to a JW algebra acting irreducibly on some Hilbert space [11]. Suppose that $c\left(\varphi_{1}\right) A^{* *}, \ldots, c\left(\varphi_{k}\right) A^{* *}$ are exceptional while $c\left(\varphi_{k+1}\right) A^{* *}$, $\ldots, c\left(\varphi_{n}\right) A^{* *}$ are JW factors. We denote by $\psi_{1}$ and $\psi_{2}$ the sum of inequivalent representations, one from each equivalence class of the sets $\left\{\pi_{1}, \ldots, \pi_{k}\right\}$ and $\left\{\pi_{k+1}, \ldots, \pi_{n}\right\}$, respectively. By Lemmas 2.1 and 2.2 ,

$$
\overline{\psi_{1}(A)}=\overline{\psi_{1}\left(\bigcap_{j=k+1}^{n} \operatorname{Ker} \pi_{j}\right)} \text {. }
$$

Since $\overline{\psi_{1}(A)}=\psi_{1}(A)\left(\psi_{1}(A)\right.$ has finite dimension) we can always find an element $x \in \bigcap_{j=k+1}^{n} \operatorname{Ker} \pi_{j}$ such that

$$
\psi_{1}(x)=\sum_{i=1}^{k} \frac{1}{2^{i}} s\left(\varphi_{i}\right)
$$

Each state $\varphi_{i}(i=1, \ldots, k)$ is multiplicative on the algebra generated by $x$. Indeed, fix $1 \leq i \leq k$, and an integer $m$. Since $s\left(\varphi_{i}\right) \leq c\left(\psi_{1}\right)$ we get

$$
U_{s\left(\varphi_{i}\right)}\left(x^{m}\right)=U_{s\left(\varphi_{i}\right)}\left(c\left(\psi_{1}\right) \circ x^{m}\right)=U_{s\left(\varphi_{i}\right)}\left(\sum_{j=1}^{k} \frac{1}{2^{j m}} s\left(\varphi_{j}\right)\right)=\frac{1}{2^{i m}} s\left(\varphi_{i}\right),
$$

and, in turn

$$
\varphi_{i}\left(x^{m}\right)=\varphi_{i}(x)^{m}
$$

Every representation $\pi_{i}$, where $i>k$, acts irreducibly on some Hilbert space $H_{i}$. (Equivalent representations will have the same Hilbert space.) Take unit vectors $\xi_{k+1}, \ldots, \xi_{n}$ from the corresponding Hilbert spaces such that

$$
\xi_{i} \in s\left(\varphi_{i}\right)\left(H_{i}\right) \quad \text { for all } i=k+1, \ldots, n \text {. }
$$

Applying now arguments in the proof of the transitivity theorem for JC algebras [14, Th. 5.2] on each Hilbert space $H_{i}$, we infer that there is an element $y \in A$ such that

$$
\psi_{2}(y) \xi_{i}=\frac{1}{2^{i}} \xi_{i} \quad \text { for all } i=k+1, \ldots, n .
$$

Moreover, in view of Lemmas 2.1 and 2.2 , we can take $y$ to be an element of the Jorclan ideal $\bigcap_{j=1}^{k} \operatorname{Ker} \pi_{j}$. As

$$
\left\langle\pi_{i}(a) \xi_{i}, \xi_{i}\right\rangle=\varphi_{i}(a) \text { for all } a \in A \text { and } i=k+1, \ldots, n,
$$

for any integer $m$ we get

$$
\varphi_{i}\left(y^{m}\right)=\left\langle\pi_{i}(y)^{m} \xi_{i}, \xi_{i}\right\rangle=\left\langle\frac{1}{2^{i m}} \xi_{i}, \xi_{i}\right\rangle=\frac{1}{2^{i m}}=\varphi_{i}(y)^{m} .
$$

In other words, the states $\varphi_{i}$ are multiplicative on the algebra generated by $y$. Put now $a=x+y$. By the previous reasoning all the states $\varphi_{1}, \ldots, \varphi_{n}$ are multiplicative on the subalgebra generated by $a$, and

$$
\varphi_{i}(a)=1 / 2^{i} \quad \text { for all } i=1, \ldots, n \text {. }
$$

Choose continuous functions $0 \leq f_{i} \leq 1(i=1, \ldots, n)$ on the real line with mutually disjoint supports and satisfying

$$
f_{i}\left(1 / 2^{i}\right)=1 \quad \text { for all } i=1, \ldots, n .
$$

By putting $a_{i}=f_{i}(a)$ we get norm one, pairwise orthogonal elements satisfying $\varphi_{i}\left(a_{i}\right)=1$ for all $i=1, \ldots, n$. The proof is complete.

Let us remark that Proposition 2.3 does not hold for infinitely many orthogonal pure states in general. This can be demonstrated by the following examples. Let $A=C_{\mathbb{E}}(X)$ be the algebra of all continuous real-valued functions on a compact Hausdorff space $X$. Then a sequence of pure states corresponding to point evaluations at distinct points $x, x_{1}, x_{2}, \ldots$, where $x$ is a limit point of $\left(x_{n}\right)$, has no supporting sequence. On the other hand, 
an essentially non-associative counterexample can be given by considering the self-adjoint part $A$ of a $C^{*}$-algebra $\mathcal{A}$ acting irreducibly on a separable Hilbert space $H$ and having zero intersection with the algebra of compact operators on $H$. Then a sequence $\left(\omega_{\xi_{n}}\right)$ of vector states corresponding to an orthonormal basis $\left(\xi_{n}\right)$ of $H$ has no supporting sequence for otherwise this sequence would consist of one-dimensional projections.

The stated examples indicate the necessity of assuming that the given states are separated in some topological way. For that reason, we introduce the following notion. Let $\tau: P(A) \rightarrow \operatorname{Prim}(A): \varphi \mapsto \operatorname{Ker} \pi_{\varphi}$ be the canonical mapping. We say that the set $P \subset P(A)$ is almost separated if there is a disjoint open covering $\left(U_{\alpha}\right)$ of $\tau(P)$ in $\operatorname{Prim}(A)$ such that each open set $\tau^{-1}\left(U_{\alpha}\right)$ contains at most finitely many elements of $P$. Observe that almost separated families of pure states have finite equivalence classes.

2.4. THEOREM. Any sequence $\left(\varphi_{n}\right)$ of almost separated mutually orthogonal pure states on $A$ has a supporting sequence.

Proof. Let $t_{n}=\tau\left(\varphi_{n}\right)$. Suppose that $\left(U_{n}\right)$ is a covering of $\left(t_{n}\right)$ obeying the conditions stated in the definition of almost separated sets. Consider the finite set $\Gamma_{1}=\left(\varphi_{n}\right) \cap \tau^{-1}\left(U_{1}\right)$. By rearranging the sequence $\left(\varphi_{n}\right)$ appropriately we can assume that $\Gamma_{1}=\left\{\varphi_{1}, \ldots, \varphi_{k}\right\}$. The set $\tau\left(\Gamma_{1}\right)$ is disjoint from the closure of $\tau\left(\left(\varphi_{n}\right)_{n>k}\right)$, whence

$$
\operatorname{Ker} \pi_{i} \not \supset \bigcap_{j=k+1}^{\infty} \operatorname{Ker} \pi_{j} \quad \text { for all } i=1, \ldots, k \text {, }
$$

where $\pi_{i}$ is the representation corresponding to $\varphi_{i}$. Applying now Proposition 2.3 for the hereditary subalgebra $\bigcap_{j=k+1}^{\infty} \operatorname{Ker} \pi_{j}$, we can find positive, norm one, pairwise orthogonal elements $a_{1}, \ldots, a_{k}$ such that

$$
\begin{array}{ll}
\varphi_{i}\left(a_{i}\right)=1 & \text { for all } i=1, \ldots, k, \\
\pi_{j}\left(a_{i}\right)=0 & \text { for all } i=1, \ldots, k \text { and } j>k .
\end{array}
$$

It follows that $\varphi_{i}\left(a_{j}\right)=\delta_{i j}$ for all $i=1, \ldots, k$ and $j=1,2, \ldots$ Proceeding analogously for the sets $\tau^{-1}\left(U_{2}\right) \cap\left(\varphi_{n}\right), \tau^{-1}\left(U_{3}\right) \cap\left(\varphi_{n}\right), \ldots$, we complete the proof.

A sequence $\left(\varphi_{n}\right)$ of orthogonal pure states on the associative algebra $C_{\mathbb{R}}(X)$ has a supporting sequence if and only if the states $\varphi_{n}$, viewed as points in the primitive ideal space $X$ of $C_{\mathbb{R}}(X)$, can be separated by open sets. Led by this example, we can state the following characterization of orthogonal pure states with supporting systems which will be useful in the sequel.

2.5. Proposition. Let $\left(\varphi_{\alpha}\right)$ be a system of pairwise orthogonal pure states on $A$. Then $\left(\varphi_{\alpha}\right)$ admits a supporting system if and only if there is a system $\left(p_{\alpha}\right)$ of open, pairwise orthogonal, projections in $A^{* *}$ such that $s\left(\varphi_{\alpha}\right) \leq p_{\alpha}$ for all $\alpha$.

Proof. Suppose $\left(a_{\alpha}\right)$ supports $\left(\varphi_{\alpha}\right)$. Then we can set $p_{\alpha}=r\left(a_{\alpha}\right)$ to get the desired orthogonal system of separating open projections.

Conversely, let $\left(p_{\alpha}\right)$ be a system of orthogonal open projections in $A^{* *}$ with $s\left(\varphi_{\alpha}\right) \leq p_{\alpha}$. Each state $\varphi_{\alpha}$ restricts to a pure state on the hereditary algebra $A_{\alpha}=U_{p_{\alpha}}\left(A^{* * *}\right) \cap A$. Therefore we can find a positive, norm one, element $a_{\alpha} \in A_{\alpha}$ with $\varphi_{\alpha}\left(a_{\alpha}\right)=1$. (This can be seen e.g. as a very special case of Proposition 2.3.) Since $a_{\alpha} \leq p_{\alpha}$ the system $\left(a_{\alpha}\right)$ is orthogonal and the implication follows.

3. Determining systems. In the previous section we proved the existence of a supporting sequence $\left(a_{n}\right)$ of a family $\left(\varphi_{n}\right)$ of almost separated orthogonal pure states. In this part we concentrate on the question whether, in addition, $\left(a_{n}\right)$ can be taken as being determining for $\left(\varphi_{n}\right)$. Closely related to this question is the problem of the existence of an associative subalgebra $B$ such that each $\varphi_{n}$ is a unique extension of some pure state on $B$. For studying these problems the following concept appears to be useful. We say that a norm one element $a$ in $A$ is a determining element for a given pure state $\varphi$ on $A$ if $\varphi$ is the only (pure) state on $A$ attaining value one at $a$. Note that in that case $\varphi(a \circ b)=\varphi(a) \varphi(b)$ for all $b \in A$. In particular, $\varphi$ is a unique extension of a pure state on the subalgebra generated by its determining element. A supporting system $\left(a_{\alpha}\right)$ of a system $\left(\varphi_{\alpha}\right)$ of mutually orthogonal pure states is called determining if each $a_{\alpha}$ is determining for $\varphi_{\alpha}$. It turns out that for separable algebras, any supporting sequence can be modified to be determining.

3.1. Proposrmion. Let $A$ be separable. Any sequence of mutually orthogonal pure states on $A$ admitting a supporting sequence has a determining supporting sequence.

Proof. Assume $\left(\varphi_{n}\right)$ is a sequence of mutually orthogonal pure states on $A$ with a supporting sequence $\left(u_{n}\right)$. Since the open projections $r\left(u_{n}\right)$ and $1-s\left(\varphi_{n}\right)$ operator commute $\left(s\left(\varphi_{n}\right) \leq r\left(u_{n}\right)\right)$ their products $r\left(u_{n}\right) \circ$ $\left(1-s\left(\varphi_{n}\right)\right)$ are open. As $A$ is separable, each hereditary subalgebra $A_{n}=$ $U_{r\left(u_{n}\right) \circ\left(1-s\left(\varphi_{n}\right)\right)}\left(A^{* *}\right) \cap A$ contains a strictly positive element $0 \leq x_{n} \leq 1$. Our goal is to prove that the elements $a_{n}=u_{n}-U_{x_{n}}\left(u_{n}\right)$ are orthogonal and determining for the states $\varphi_{n}$. The orthogonality of $\left(a_{n}\right)$ follows from the orthogonality of the projections $r\left(u_{n}\right)$. Also, $a_{n} \leq u_{n} \leq 1$ and $a_{n} \geq-U_{x_{n}}\left(u_{n}\right) \geq-U_{x_{n}}(1)=-x_{n}^{2} \geq-1$ gives $\left\|a_{n}\right\| \leq 1$. Since $\mathcal{L}_{\varphi_{n}}=$ $U_{1-s\left(\varphi_{n}\right)}\left(A^{* *}\right) \cap A$ is a quadratic ideal containing $x_{n}$ we have $U_{x_{n}}\left(u_{n}\right) \in \mathcal{L}_{\varphi_{n}}^{+}$, whence $\varphi_{n}\left(U_{x_{n}}\left(u_{n}\right)\right)=0$. Consequently, $\varphi_{n}\left(a_{n}\right)=1$ for each $n$. 
It remains to prove that $a_{n}$ is determining for $\varphi_{n}$. For this, first observe that $r\left(x_{n}\right)=r\left(u_{n}\right) \circ\left(1-s\left(\varphi_{n}\right)\right)$. Indeed, suppose that $r\left(x_{n}\right)<$ $r\left(u_{n}\right) \circ\left(1-s\left(\varphi_{n}\right)\right)$. Then there exists a normal state $\psi$ on the algebra $U_{r\left(u_{n}\right) \circ\left(1-s\left(\varphi_{n}\right)\right)}\left(A^{* *}\right)$ with $\psi\left(r\left(x_{n}\right)\right)=0$. By the Schwarz inequality $\psi\left(x_{n}\right)$ $=0$ and the strict positivity of $x_{n}$ entail that $\psi$ vanishes on $A_{n}$. Since $r\left(u_{n}\right) \circ\left(1-s\left(\varphi_{n}\right)\right)$ is in $\bar{A}_{n}$ we get $\psi\left(r\left(u_{n}\right) \circ\left(1-s\left(\varphi_{n}\right)\right)\right)=0$, a contradiction.

Take now a pure state $\varrho$ on $A$ with $\varrho\left(a_{n}\right)=1$. The inequalities

$$
0 \leq \varrho\left(u_{n}\right), \varrho\left(U_{x_{n}}\left(u_{n}\right)\right) \leq 1
$$

yield $\varrho\left(u_{n}\right)=1$ and $\varrho\left(U_{x_{n}}\left(u_{n}\right)\right)=0$. The functional $\varrho\left(U_{x_{n}}(\cdot)\right)$ being normal on $A^{* *}$, we find that also $\varrho\left(U_{x_{n}}\left(r\left(u_{n}\right)\right)\right)=0$. As $x_{n}, x_{n}^{2} \in U_{r\left(u_{n}\right)}\left(A^{* *}\right)$ we have $U_{x_{n}}\left(r\left(u_{n}\right)\right)=x_{n}^{2} \circ r\left(u_{n}\right)=x_{n}^{2}$. In other words, $\varrho\left(x_{n}^{2}\right)=0$ and so $\varrho\left(x_{n}\right)=0$. Therefore $\varrho\left(r\left(x_{n}\right)\right)=\varrho\left(r\left(u_{n}\right) \circ\left(1-s\left(\varphi_{n}\right)\right)\right)=0(\varrho$ is normal on $\left.A^{* *}\right)$, which implies $\varrho\left(r\left(u_{n}\right)\right)=\varrho\left(s\left(\varphi_{n}\right)\right)$. But $\varrho\left(u_{n}\right)=1$ and immediately $\varrho\left(r\left(u_{n}\right)\right)=1$. Hence $\varrho\left(s\left(\varphi_{n}\right)\right)=1$, implying $\varrho=\varphi_{n}$. It can be observed easily that the positive part of a determining element is again determining for a given state. Thus, passing to the positive parts, we can assume all $a_{n}$ 's to be positive, which concludes the proof.

Combining now Theorem 2.4 and Proposition 3.1, we get the following result:

3.2. THEOREM. Let $\left(\varphi_{n}\right)$ be a sequence of almost separated, mutually orthogonal pure states on a separable algebra $A$. Then $\left(\varphi_{n}\right)$ admits a determining supporting sequence. In particular, there is a maximal associative subalgebra $B$ of $A$ such that each restriction $\varphi_{n} \mid B$ is a pure state of $B$ which extends uniquely to a state of $A$.

Proof. Take a determining supporting sequence $\left(a_{n}\right)$ of $\left(\varphi_{n}\right)$ whose existence is guaranteed by Theorem 2.4 and Proposition 3.1. Let $C$ be the subalgebra generated by the elements $\left(a_{n}\right)$. Then $C$ is associative and any state $\varphi_{n}$ is uniquely determined by its pure restriction to $C$. Since any pure state extends from $C$ to a pure state on an arbitrary larger algebra, it can be easily seen that any maximal associative subalgebra $B$ of $A$ containing $C$ will satisfy the statement of the theorem. The proof is complete.

As a corollary of the previous theorem, we deduce that any finite family $\varphi_{1}, \ldots, \varphi_{n}$ of pairwise orthogonal pure states on a separable algebra $A$ is uniquely determined by the pure state restrictions $\varphi_{1}\left|C, \ldots, \varphi_{n}\right| C$, where $C$ is some associative subalgebra generated by $n$ orthogonal elements. As every algebra generated by two orthogonal elements is generated by their sum, we can add that every pair of orthogonal pure states on a separable algebra is uniquely given by their values on some singly generated (i.e. smallest possible) subalgebra. This generalizes [2, Theorem].
4. Supporting sequences and dual algebras. In this section we characterize dual JB algebras in terms of determining supporting systems and answer the question: For what algebras does every orthogonal system of states have a determining supporting system?

Recall that a JB algebra $A$ is called dual if $\left(I^{0}\right)^{0}=I$ for every norm closed quadratic ideal of $A$, where the annihilator of a set $S \subset A$ is defined to be $S^{0}=\{a \in A \mid a \circ s=0$ for all $s \in S\}$. It is well known that dual $C^{*}$-algebras are nothing but algebras of compact operators. The structure theory of dual JB algebras has been developed in [7]. We say that $A$ is $s$ monotone closed if it is closed with respect to infima of decreasing sequences of positive operator commuting elements.

4.1. Theorem. Let $A$ be a JB algebra. The following statements are equivalent:

(i) $A$ is dual.

(ii) For every pure state $\varphi$ on $A$ there is a projection $p \in A$ which is determining for $\varphi$.

(iii) $A$ is $s$-monotone closed and every pure state on $A$ has a determining element.

(iv) Every system of mutually orthogonal pure states on $A$ has a determining supporting system.

Proof. (i) $\Rightarrow$ (ii). If $A$ is dual, then $A$ contains all minimal projections in $A^{* *}$ (see [7]). Thus, $s(\varphi) \in A$ is a determining projection for each pure state $\varphi$.

(ii) $\Rightarrow$ (i). Assume $p$ is a determining projection for $\varphi$. Then $p=s(\varphi)$. Indeed, it is clear that $s(\varphi) \leq p$. If the projection $p-s(\varphi)$ were non-zero, then there would be a normal state $\psi$ on $A^{* *}$ with $\psi(p-s(\varphi))=1$. Therefore, $\psi$ would give a state on $A$ different from $\varphi$ such that $\psi(p)=1$, a contradiction. In other words, $A$ contains all minimal projections in $A^{* *}$ and is therefore dual by [7].

(i) $\Rightarrow$ (iii). This follows from the implication (i) $\Rightarrow$ (ii) and the fact that each maximal associative subalgebra of a dual algebra has discrete spectrum, and it is therefore monotone closed.

(iii) $\Rightarrow$ (i). It suffices to prove that any pure state $\varphi$ on $A$. has a projection as a determining element, and to apply the implication (ii) $\Rightarrow$ (i). Suppose $0 \leq x<1$ is a determining element for a state $\varphi$. The sequence $\left(x^{n}\right)$ of its powers is decreasing, and so it has a limit $a \in A$. As $a \leq x$, we have $\varrho(a)<1$ for any state $\varrho$ different from $\varphi$. Since $\varphi(a)=1$, it follows that $a$ is a determining element for $\varphi$. Consider now the associative subalgebra $C$ of $A$ generated by $x$. For any pure state $\varrho$ on $C$ which does not coincide with the restriction of $\varphi$ to $C$, we have $\varrho(x)<1$ and so $\varrho(a)=0$. Thus, $a$ is a projection by spectral theory. 
(i) $\Rightarrow$ (iv). This is an immediate consequence of the fact that any projection in the double dual of a JB algebra is open [7], and of Proposition 2.5 .

(iv) $\Rightarrow$ (i). Let $\left(\varphi_{\alpha}\right)$ be a system of mutually orthogonal pure states such that $\sum_{\alpha} s\left(\varphi_{\alpha}\right)=z_{\text {at }}$, where $z_{\text {at }}$ is the projection in $A^{* *}$ which is the supremum of all minimal projections in $A^{* *}$. Assume that $\left(a_{\alpha}\right)$ is its determining supporting system. Then $a_{\alpha} z_{\text {at }}=s\left(\varphi_{\alpha}\right)$ for all $\alpha$. Since $a \mapsto a z_{\text {at }}$ is a faithful representation of $A$, we see that each $a_{\alpha}$ has to be a projection. Finally, employing the fact that $a_{\alpha}$ is determining for $\varphi_{\alpha}$ we conclude that $\psi\left(a_{\alpha}\right)<1$ for any normal state $\psi$ on $A^{* *}$ concentrated at $1-z_{\text {at. }}$ In other words, $\left(1-z_{\text {at }}\right) a_{\alpha}=0$ and so $a_{\alpha}=s\left(\varphi_{\alpha}\right)$. Hence, $A$ contains all minimal projections in its second dual and it is therefore dual by [7]. The proof is complete.

Every pure state on a separable algebra has automatically a determining element by Theorem 3.2. Thus, by Theorem 4.1 we see e.g. that a separable $\mathrm{JB}$ algebra is dual if and only if it is s-monotone closed.

Acknowledgements. The author would like to express his gratitude to the Alexander von Humboldt Foundation, Bonn, for supporting his research, the result of which is presented in this paper. He is also grateful to Prof. D. Kölzow, Erlangen, who acted as a scientific host during the author's stay at the Mathematical Institute, University of Erlangen, for his hospitality, helpful remarks and encouragement. He also acknowledges the support of the Grant Agency of the Czech Republic (Grant No. 201/96/0117) and Czech Technical University (Grant No. J04/98/210000010).

\section{References}

[1] J. F A Arnes and R, V. Kadison, Pure states and approximate identities, Proc. Amer. Math. Soc. 21 (1969), 749-752.

[2] C. A. Akemann, Approximate units and maximal abelian $C^{*}-$ subalgebras, Pacific J. Math. 33 (1970), 543-550.

[3] - Left ideal structure of $C^{*}$-algebras, J. Funct. Anal. 6 (1970), 305-317.

[4] C. A. Akemann, J. Anderson and G. K. Pedersen, Excising states on $C^{*}$ algebras, Canad. J. Math. 38 (1986), 1239-1260.

[5] -, -, -, Approaching infinity in $C^{*}$-algebras, J. Operator Theory 21 (1989), 255271.

[6] J. Anderson, Extensions, restrictions, and representations of states on $C^{*}$-algebras, Trans. Amer, Math. Soc. 249 (1979), 303-329.

[7] L. J. Bunce, The theory and structure of dual JB-algebras, Math. Z. 180 (1982) $525-534$.

[8] S. P. Gudder, Stochastic Methods in Quantum Mechanics, North-Holland, Amsterdam, 1979.
[9] J. Ham halter, Gleason property and extensions of states on projection logics, Bull. London Math. Soc. 26 (1994), 367-372

[10] J. Hamhalter, Determinacy of states and independence of operator algebras, Internat. J. Theoret. Phys. 37 (1998), 599-607.

[11] H. Hanche-Olsen and E. Størmer, Jordan Operator Algebras, Pitman, Boston, 1984.

[12] R. V. Kadison and I. M. Singer, Extensions of pure states, Amer. J. Math. 81 (1959), 383-400.

13] I. E. Segal, Postulates for general quantum mechanics, Ann. of Math. 48 (1947) $930-938$

[14] E Størmer, Irreducible Jordan algebras of self-adjoint operators, Trans. Amer. Math. Soc. 130 (1968), 153-166.

Department of Mathematics

Faculty of Electrical Engineering

Czech Technical University

Technicka 2

16627 Praha 6, Czech Republic

E-mail: hamhalte@math.feld.cvut.cz

Received July 20, 1998

Revised version February 18, 1999 